\title{
The role of response-reinforcer correlation in signaled reinforcement effects
}

\author{
TODD R. SCHACHTMAN \\ University of Missouri, Columbia, Missouri \\ and \\ PHIL REED \\ University of Oxford, Oxford, England
}

\begin{abstract}
In three experiments, we examined the effects of signaling reinforcement during operant responding in order to illuminate the factors underlying instrumental overshadowing and potentiation effects. Specifically, we examined whether signaling reinforcement produces an enhancement and attentuation of responding when the response-reinforcer correlation is weak and strong, respectively. In Experiment 1, rats responded on variable-ratio (VR) or variable-interval (VI) schedules that were equated for the number of responses emitted per reinforcer. A signal correlated with reinforcement enhanced response rates on the VR schedule, but attenuated response rates were produced by the signal on the VI schedule. In Experiment 2, two groups of rats responded on a VI schedule while the two other groups received a conjoint VI, negative fixed-ratio schedule in which the subjects lost the availability of reinforcements if they emitted high response rates. A reinforcement signal attenuated responding for the simple VI groups but not for the animals given the negative fixed-ratio component, although the signal improved response efficiency in both groups. In Experiment 3, a poor correlation between responding and reinforcement was produced by a VI schedule onto which the delivery of response-independent food was superimposed. A signal for reinforcement initially elevated responding on this schedule, relative to an unsignaled condition; however, this pattern was reversed with further training. In sum, the present experiments provide little support for the view that signaling reinforcement enhances responding when the response-reinforcer correlation is weak and attenuates responding when this correlation is strong.
\end{abstract}

Signaling a brief delay of reinforcement $(500 \mathrm{msec})$ on a variable-interval (VI) schedule attenuates levels of responding relative to an unsignaled control condition. In contrast, a signal-induced enhancement in response rate results if a long interval (e.g., $3 \mathrm{sec}$ ) is scheduled between the response that precedes food and food delivery (Richards, 1981; Schachtman, Reed, \& Hall, 1987; Williams \& Heyneman, 1982). Schachtman et al. (1987) interpreted these findings as indicative of the importance of the contiguity between response and reinforcement in determining when signaling reinforcement will produce an attenuation of performance (i.e., instrumental overshadowing) or an enhancement of responding (i.e., instrumental potentiation). A very brief delay of reinforcement is presumed to result in a strong response-reinforcer

\footnotetext{
This research was supported in part by a grant from the United Kingdom Science and Engineering Research Council to G. Hall, and by funds from the University of Missouri. We thank J. E. Wright, University of Leeds, for his support and the loan of equipment, and Steve Reilly and Mark Good for comments on an earlier version of this manuscript. We thank David Knipp for assisting with the preparation of the manuscript. Requests for reprints may be addressed to T. R. Schachtman, Department of Psychology, University of Missouri, Columbia, MO 65211, or P. Reed, Department of Experimental Psychology, University of Oxford, South Parks Road, Oxford OX1 3UB, England.
}

association that is weakened by the imposition of a signal during the delay. A longer delay of reinforcement presumably results in a weak response-reinforcer association, and responding is enhanced when a signal is placed during this delay. Bouton, Jones, McPhillips, and Swartzentruber (1986) have made a similar claim with regard to odor-aversion learning studies.

Although one effect of a delay of reinforcement in, for example, the Schachtman et al. (1987) report was to influence the contiguity between response and reward, the duration of the delay may also have interacted with the effects of signaling reinforcement because it influenced the correlation or contingency between responding and reinforcement. Baum (1973) suggested that changes in reinforcement delay may best be viewed as having an effect on the response-reinforcer correlation. Reinforcement delays certainly influence response-reinforcer contiguity, but the issue remains open as to whether contiguity or correlation is the critical factor underlying the interaction between reinforcement delay and signaled reinforcement. Consequently, the effects reported by Schachtman et al. and Williams and Heyneman (1982) may have been produced by manipulations in response-reinforcer correlation through the use of different reinforcement delays. The strength of a positive response-reinforcer correla- 
tion is an inverse function of the frequency of occurrences of reinforcement given no response and of a response given no reinforcement, and is a direct function of the occurrences of reinforcement given a response and, potentially, of no reinforcement given no response (i.e., Delta $P$; see Hammond \& Paynter, 1983). It is possible that signaling reinforcement produces potentiation when the response-reinforcer correlation is poor, and overshadowing when this correlation is strong.

The results reported by Reed, Schachtman, and Hall (1988a, 1988b; see also Catania \& Keller, 1981) are consistent with the correlation notion described above, in that signaling reinforcement produced potentiation when the correlation between the response and the reinforcer was poor-for example, on schedules in which a large number of responses were emitted per reinforcement. The reports by Reed et al. demonstrated that a signal-induced potentiation effect occurred when subjects were responding on a variable ratio (VR) schedule of reinforcement, and that this effect was larger when the ratio criterion was greater. Since the number of response-reinforcer pairings and the scheduled delay between the response that produced food and the reinforcer were held constant for the different groups in those studies, differences in the effect of the signal could not be attributed to those influences. One factor that did distinguish the VR schedules (i.e., VR 10 and VR 30) was the number of nonreinforced responses emitted by the subjects on the various schedules; that is, the response-reinforcer correlation was lower for animals that received the higher VR schedule criterion.

These findings lend support for the view that signaling reinforcement will enhance performance (relative to an unsignaled condition) when the response-reinforcer correlation is poor, and will retard performance when this contingency is relatively good. Moreover, the effects of signals on differential reinforcement of high (DRH) and low (DRL) rate schedules (see Tarpy \& Roberts, 1985) are also consistent with the view that correlation (e.g., the number of nonreinforced responses) is the critical factor. DRL schedules typically produce high response-reinforcer correlations (relatively few nonreinforced responses) and reinforcement signals produce instrumental overshadowing; DRH schedules involve poorer responsereinforcer correlations (a relatively large number of nonreinforced responses) and signal-induced potentiation results (Tarpy \& Roberts, 1985).

The response-reinforcer correlation notions can account for most of the existing reinforcement-signaling effects. There are a host of other theories on instrumental signaling effects, including focus on factors such as reinforcement of response bursts (e.g., Hall, Channell, \& Schachtman, 1987; Williams \& Heyneman, 1982), sign tracking (Iversen, 1981), response-reinforcer associative strength (Schachtman et al., 1987), and response efficiency (e.g., Roberts, Tarpy, \& Lea, 1984); however, none of these theories can explain all of the reported findings. In the present experiments, we sought to further examine the influence of response-reinforcer correlation on signaled reinforcement effects using a variety of schedules with the components that potentially influence the correlation between responding and reinforcement.

In Experiment 1, we examined the effects of reinforcement signals on VI and VR schedules when the number of responses per reinforcer was equated for the two schedules. In Experiment 2, we investigated such effects on VI schedules that had an additional componentscheduled response-contingent reinforcer omissionwhich produced a poor correlation between response rate and reinforcement frequency. Experiment 3 examined signaling effects on a schedule that decreased the positive response-reinforcer correlation by adding responseindependent foods.

\section{EXPERIMENT 1}

In the present experiment, we used a yoking procedure to investigate the possibility that when the number of responses emitted per reinforcer is approximately equal on VI and VR schedules, similar signaling effects occur for both schedules due to the comparable responsereinforcer correlations. Reed et al. (1988a, Experiment 2) obtained an overshadowing effect on both a VI and VR schedule, but the yoking procedure in that study (see also Dickinson, Peters, \& Shechter, 1984) introduced a confounding variable between signaled and unsignaled VR conditions. In the present experiment, we used a yoking procedure that eliminated this confound. Examining the potential for differential effects of a signal for reinforcement on VI and VR schedules when the responsereinforcement correlation is equated provides one test of the correlation account.

\section{Method \\ Subjects. Thirty-two male Lister hooded rats served as subjects. The rats were 4-6 months old at the start of the study, had an ad lib weight range of $350-415 \mathrm{~g}$, and were maintained at $80 \%$ of this weight. The animals were housed in pairs with water constantly available in the home cage. All of the subjects had served in an appetitive classical conditioning study in which food pellets were paired with the illumination of a jewel light, but they were naive with respect to leverpressing and the visual stimulus used in the present experiment.}

Apparatus. Four identical operant chambers (Campden Instruments Ltd.) were employed. Each chamber was housed inside a light- and sound-attenuating enclosure. A background masking noise (65 dBA re SPL) was supplied by a ventilating fan. Each chamber was equipped with two retractable levers, one of which was withdrawn and inoperative throughout the study. The food tray into which reinforcement (one 45-mg Noyes food pellet) could be delivered was centrally located between the two levers. The ceiling of the chamber was a white translucent Perspex panel, which allowed diffuse illumination to be delivered by a 30-W fluorescent strip light mounted above the ceiling. While counterbalancing the animals on three response rates, the rats were also counterbalanced, to the extent possible, as a function of their treatment in the previous study.

Procedure. Because of the animals' previous experience, they needed no magazine training. Pretraining commmenced with two sessions in which every leverpress was reinforced (CRF). Each ses- 
sion continued until the animal had earned 75 reinforcements. Subsequently, three 40-min sessions were given in which a VI 30-sec (range 1-90 sec) schedule of reinforcement occurred. Following this treatment, the animals were divided into four groups $(n=8)$ that were counterbalanced for response rate on the final session of VI 30.

Two groups of rats received reinforcement according to a VI 60 $\mathrm{sec}$ schedule (range 2-180 sec). One of these groups received a flash of diffuse light delivered from the strip light during a 500-msec delay between the criterion response and reinforcement. The average number of responses emitted per reinforcer over the session by all 16 animals responding on the VI schedule determined the VR value for both groups of subjects receiving reinforcement according to the VR schedule. That is, the total number of responses emitted by the VI subjects was divided by the total number of reinforcements earned by those subjects. This schedule avoided the confounds potentially present in previous yoking procedures that yoked individual animals from different signaling conditions (see Dickinson et al., 1984; Reed et al., 1988a). This score was then used as the nominal VR value for all yoked subjects (ranging from one to two times the mean VR value). As with the VI subjects, one group of rats receiving the VR schedule had a 500 -msec delay of reinforcement signaled by the flash of diffuse light, whereas the other VR group received unsignaled delayed reinforcement, a control condition used by Pearce and Hall (1978, Experiment 3). Sixteen sessions of this treatment were given. All sessions lasted until 40 reinforcers had been obtained.

\section{Results and Discussion}

The rates of responding during the final session of pretraining for the two groups that were to experience the yoked VR schedule were 9.78 responses per minute for the to-be-signaled group, and 8.39 responses per minute for the to-be-unsignaled group. The rates of responding for the groups that were to receive the corresponding treatments on the master VI schedule were 10.22 and 8.49 responses per minute, respectively. An analysis of variance (ANOVA) conducted on these data revealed no significant differences between the groups $(F \mathrm{~s}<1)$.

The group mean rates of responding are displayed as two-session blocks in Figure 1. Inspection of the data reveals that the subjects receiving the VR schedule responded at a higher rate than did the subjects receiving the VI schedule. The effects of the reinforcement signal depended upon the schedule. Attenuated rates of responding were produced by the signal in the VI subjects relative to the corresponding control condition, but a signalinduced enhancement of responding was generated on the VR schedule. This description of the results was corroborated by a three-factor ANOVA (signal $x$ schedule $\times$ block) conducted on the response rates, which revealed an interaction of all three factors $[F(7,196)=5.92$, $p<.01]$.

Due to this interaction, the effect of the reinforcement signal on the two schedules was analyzed separately by two-factor ANOVAs (signal $\times$ block). The ANOVA conducted upon the VR subjects' performance revealed a significant signal $\times$ block interaction $[F(7,98)=6.32$, $p<.01]$, indicating that the group receiving the signal acquired responding to a greater extent than did the unsignaled group. The ANOVA conducted on the VI groups

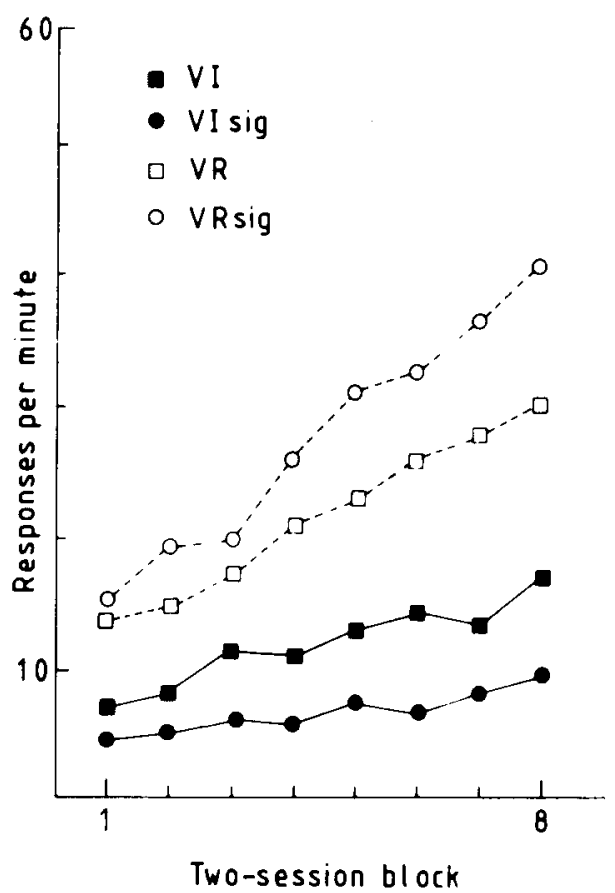

Figure 1. Results from Experiment 1. Mean response rates for the four groups, represented as two-session blocks. VI = variableinterval schedule, $\mathrm{VR}=$ variable-ratio schedule, sig = signaled reinforcement.

revealed a main effect of signal $[F(1,14)=10.75$, $p<.01]$ and of block $[F(7,89)=7.31, p<.01]$, but no interaction between these two factors $(p>.05)$. Thus, on the VI schedule, the unsignaled condition yielded higher response rates than did the signaled reinforcement condition.

The present results offer no support for the view that the greater number of responses required for reinforcement by a VR schedule, relative to a VI schedule, produces differential effects of a reinforcement signal. The number of responses per reinforcement were held constant for VI and VR schedules, yet the signal produced overshadowing in the former case and potentiation in the latter case. These findings confirm the effects obtained by Reed et al. (1988a, Experiments 1 and 3) with respect to the effects of a signal on VR and VI schedules, although the conclusions based on the present findings may require qualification in that nonnaive rats were used as subjects. However, it is worth noting that we have obtained similar effects on such schedules (albeit without the yoking procedure) in a previous study using rats that were experimentally naive to cues of the modality used for the signal (e.g., Reed et al., 1988b).

It is apparent that the correlation view of the effects of a signal for reinforcement cannot account for the present results. The present findings are, however, consistent with the view that a signal for reinforcement will enhance an animal's detection of the specific requirement of the schedule-that is, spacing responses appropriately 
for the VI schedule (Roberts et al., 1984) or behavior appropriate for maximizing the number of reinforcements per unit of time on the VR schedule.

\section{EXPERIMENT 2}

To further test the correlation view, Experiment 2 was designed to investigate the effects of a reinforcement signal on responding on a schedule that included a component with a negative relationship between response rate and reinforcement frequency. Vaughan and Miller (1984) developed an instrumental schedule of reinforcement that organized a "negative feedback" between response rate and reinforcement frequency. This schedule was arranged so that, as response rates increased beyond a certain level, reinforcement frequency decreased. Animals, although sensitive to this contingency (Ettinger, Reid, \& Staddon, 1987; Reed \& Schachtman, 1989; Vaughan, 1982), demonstrate extremely inefficient patterns of responding (Vaughan \& Miller, 1984), which result in a very poor response-reinforcer correlation. If potentiation is likely when the response-reinforcer correlation is low, then such an effect should be observed on a schedule with a component in which responses can produce reinforcement omission. That is, this negative feedback schedule has been shown to produce a large number of responses per reinforcer earned, relative to a normal VI schedule. Hence, the schedule results in a poorer response-reinforcer correlation. This poor correlation would allow signal-induced potentiation of instrumental responding, according to the correlation view. Such a poor correlation would be expected to make this contingency particularly sensitive for producing a signal-induced enhancement in response rate, as would be expected on the basis of the correlation notion.

\section{Method}

Subjects. Thirty-two experimentally naive male hooded rats (PVG) served as subjects. The rats were 4-6 months old at the start of the study and had an ad lib weight range of $350-420 \mathrm{~g}$. The animals were maintained as described in Experiment 1.

Apparatus. Four operant chambers, identical to those described in Experiment 1 except that they had a transparent Perspex ceiling, were employed in the present experiment. A speaker was located on the side of the chamber through which a 100-dBA tone (35 dBA above background) could be presented. No houselight was used in this experiment.

Procedure. The subjects were magazine trained by exposure to two 30-min sessions of a variable time (VT) 60-sec schedule. During both of these sessions, the levers were withdrawn from the chamber. In the first session, the flaps covering the recessed food tray were taped open to allow easy access to the food pellets. In all subsequent sessons, the flaps were lowered to their resting position. Following magazine training, the animals were trained to leverpress as in Experiment 1. Once leverpressing was established, two 40-min sessions of a VI 30-sec schedule with immediate reinforcement were given. Following VI 30 pretraining, the subjects were given two sessions of a VI 60-sec schedule with immediate reinforcement. The subjects were then assigned to one of four groups $(n=8)$, which were counterbalanced for response rate and response efficiency (i.e., responses per reinforcer) during the two sessions of VI 60-sec training.
Two of the groups received the negative feedback contingency schedule: a conjoint VI 60 negative fixed-ratio (FR) 30 schedule. One of these groups (Group VI-FR sig) received a 500-msec signal, a 100-dBA tone during a 500-msec delay of reinforcement; the other group (Group VI-FR unsig) received unsignaled delayed reinforcement. All of these subjects responded on a conjoint schedule such that the occurrence of reinforcement was determined by a VI 60 schedule (range 1-180 sec). When an interval timed out, a reinforcer became available and was stored in a "bank" until the next response was emitted. The timer controlling the VI 60 schedule continued to run whether or not a response occurred. The second component of the conjoint schedule consisted of a negative FR 30 (-FR 30) schedule. That is, a reinforcer was subtracted from the bank every time the FR value of 30 responses was satisfied. As additional reinforcers became available, they were added to those already stored in the bank; however, only one food pellet was delivered at a time. The bank could potentially become negative if the subject responded at a high rate, and food would not be delivered until the bank contained at least one reinforcer.

To summarize, the animals perform on a VI 60 schedule, and each time an interval timed out, one reinforcer was added to the bank. If an animal failed to respond during a period when two or more intervals timed out, then the bank could be greater than 1 (e.g., 2 ) and the next response would produce one pellet. The bank would then remain at 1 , and another interval would have to time out for a response to produce reinforcement. Hence, if the bank was greater than one, then responding did not result in CRF until the bank equaled zero. Each time the animal completed 30 responses, one reinforcer was subtracted from the bank.

The remaining two groups (VI sig and VI unsig) received only the VI 60 schedule component. One of these groups (VI unsig) received an unsignaled 500 -msec delay of reinforcement; the other group (VI sig) received the same signaling treatment as Group VI-FR sig. The experiment continued for 20 sessions; each session lasted $40 \mathrm{~min}$.

\section{Results and Discussion}

The left panel of Figure 2 shows the mean response rates for all four groups, represented as four-session blocks. From inspection of the data, it is clear that, as expected, the signal attenuated the response rate for the animals on the simple VI schedule relative to that of the simple VI subjects that received unsignaled reinforcement. This effect, however, was not as pronounced in the group that experienced signaled reinforcement on the negative feedback schedule (compare Groups VI-FR sig and VI-FR unsig). A three-factor ANOVA (contingency $x$ signal $\times$ block) conducted on the response rates displayed in Figure 2 revealed a statistically significant three-way interaction $[F(4,112)=3.08, p<.01]$.

Due to the interaction, the two contingency schedules (VI and VI-FR) were analyzed separately using two-factor ANOVAs (signal $\times$ block). The ANOVA conducted on the data from the simple VI groups revealed that the signal produced a statistically significant decrease in response rate $[F(1,14)=20.72, p<.001]$. There was also a significant main effect of block $[F(4,56)=3.32, p<.01]$, but the interaction of the two factors was not significant $(p>.10)$. The two-factor ANOVA (signal $\times$ block) conducted on the response rates produced by the negative feedback conditions revealed a significant interaction between these two factors $[F(4,56)=3.26, p<.01]$. Analysis of the simple main effects for the negative feedback 


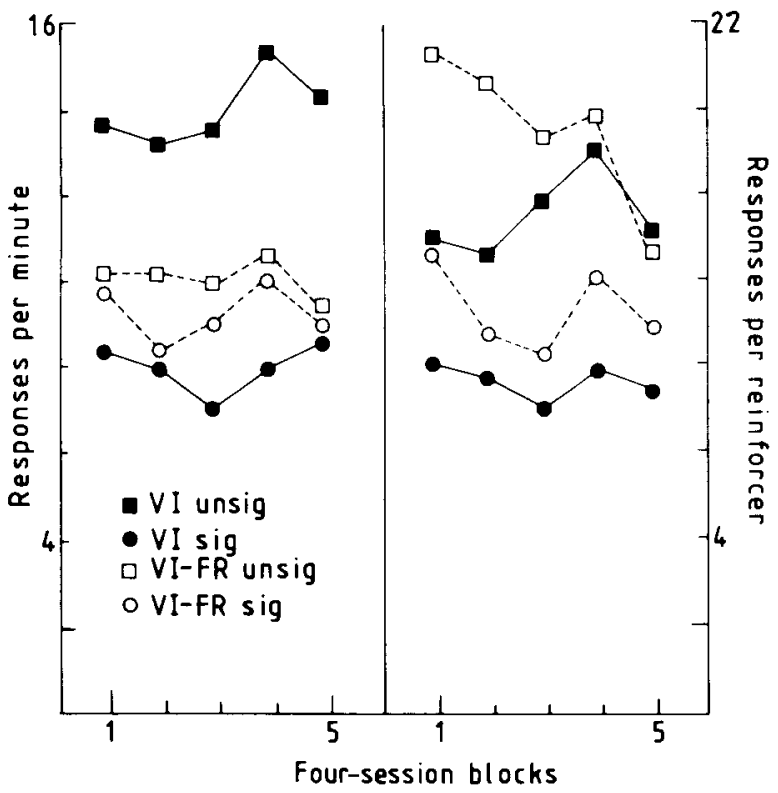

Figure 2. Results from Experiment 2. Left panel: Mean response rates for the four groups, represented as four-session blocks. Right panel: Mean efficiency scores for all four groups, represented as four-session blocks. VI = simple variable-interval schedule, VI-FR = negative feedback schedule, sig = signaled reinforcement, unsig $=$ unsignaled reinforcement.

groups demonstrated that only on Block 2 did the signal produce a lower response rate than in the unsignaled group $(p<.05)$, indicating a smaller effect of the signal on this schedule relative to the simple VI schedule. Although response rates for the VI-FR conditions were low, a floor effect was unlikely since lower rates could have occurred (6 of the 8 subjects in Group VI-FR sig consistently responded above 8 responses per minute). Moreover, the present group means are comparable to the response rates of previous studies examining the effects of signaled reinforcement during instrumental responding (e.g., Pearce \& Hall, 1978, Experiment 1; Roberts et al., 1984, Experiment 1; Tarpy \& Roberts, 1985, Experiment 1).

The right panel of Figure 2 shows the group mean number of responses per reinforcer represented as four-session blocks. Considering response rates and efficiency together, it can be seen that the two VI-FR groups yielded comparable scores on the former measure but differed on the latter measure. This may have occurred because a modest difference between the groups in the total responses emitted per session might not greatly influence response rate differences but might have a large effect on efficiency, since fewer food pellets for one group would be earned. This was especially true for animals that obtained very few food pellets due to very high response rates. Over the course of the present experiment, Group VI-FR sig earned a mean of 30 reinforcers per session, whereas Group VI-FR unsig earned a mean of 24 per session, a difference that was statistically significant $[F(1,14)$ $=24.71, p<.01]$.

Comparison of the unsignaled groups in each schedule condition revealed that the subjects that experienced the negative feedback contingency initially emitted more responses per reinforcer than did the subjects that performed on the simple VI schedule. That is, the animals that experienced the former condition were less efficient than those responding on the simple VI schedule. This is not altogether surprising, since the VI-FR groups received fewer reinforcements, owing to the negative feedback contingency. Over the course of training, however, the number of responses emitted per reinforcer by Group VI-FR unsig decreased. Indeed, by the last block, the efficiency measure for this group was comparable to that of Group VI unsig. Hence, although the correlational account of reinforcement-signaling effects would not predict differential signaling effects beyond Session 12 , the absence of a potentiation effect with the VI-FR schedule earlier in training is not very supportive of the correlational view. The reinforcement signal improved the subjects' efficiency in both the simple VI and the negative feedback groups, relative to their corresponding unsignaled groups. A three-factor ANOVA (contingency $\times$ signal $\times$ block), conducted on the efficiency scores depicted in the right panel of Figure 2 revealed statistically significant effects of each of the factors, ( $p s<.01)$. The interaction between contingency and block just missed the level of significance $[F(4,112)=$ $3.41, .06>p>.05]$ and no other interaction approached significance $(p s<.05)$.

The response rates generated by the signal on the negative schedule do not lend particularly convincing support for the correlation view of signaled reinforcement effects. The inefficient performance produced by the VI-FR schedule caused a large number of nonreinforced responses and resulted in a poor contingency between responding and reinforcement. The correlation view of signaling effects predicts potentiation on this schedule. However, the attenuated overshadowing effect on VI-FR schedules, relative to the VI schedules, does not allow complete dismissal of the correlational account.

It is also worth noting that the difference in magnitude of the overshadowing effects on the two schedules occurred primarily because of differences in response rates of the two unsignaled conditions. A similar finding was obtained by Schachtman et al. (1987, Experiment 1) when potentiation and overshadowing were observed in the same sessions using a four-component multiple schedule, and it was this result that initially kindled the correlational explanation of signaling effects.

\section{EXPERIMENT 3}

In the previous two experiments, we tested the correlation view by manipulating the probability of a reinforcer given a response (i.e., the number of nonreinforced responses). The correlation between responding and reinforcement can also be altered by manipulating the probability of reinforcement in the absence of responding. In Experiment 3, we used a schedule that was expected to produce a poor response-reinforcer correlation by imposing a VT 30-sec component on the VI 60-sec base schedule. In classical conditioning, degrading the contingency 
between a CS and a US can be achieved by scheduling the US to occur during the intertrial interval (e.g., Gibbon \& Balsam, 1981; Kasprow, Schachtman, \& Miller, 1987; Rescorla, 1968). A similar procedure in which response-independent reinforcements were scheduled in order to weaken the correlation between responding and reinforcement in instrumental learning was found to decrease response rates (e.g., Dickinson \& Charnock, 1985; Hammond, 1980; Rachlin \& Baum, 1972). This method of degrading the correlation may be a more appropriate test of the correlation account of signaling effects than the comparison of VI-FR and simple VI schedules of Experiment 2, since it does not explicitly involve the omission of response-reinforcer pairings relative to simple VI schedules.

\section{Method}

Subjects and Apparatus. Sixteen male Lister hooded rats served in this experiment. The subjects had previously been used in a conditioned suppression of leverpressing study in which they had experienced presentations of diffuse lights, white noise, and footshock. They were, however, naive with respect to the auditory stimulus, a tone, used in the present study. The apparatus was identical to that described in Experiment 2.

Procedure. The animals were divided into two groups $(n=8)$, counterbalanced for experience and performance on the final session of operant baseline training in their previous classical conditioning experiment. Responding was maintained in the present experiment by a VI $60-\mathrm{sec}$ schedule with a $500-\mathrm{msec}$ delay of reinforcement. Response-independent food pellets were delivered on a VI 30-sec schedule (range $2-90 \mathrm{sec}$ ). This rate of noncontingent reinforcement would be fully expected to attenuate response rates given that, on average, two response-independent food presentations occurred for every response-contingent food reinforcement. One group of animals had reinforcement signaled by a $100-\mathrm{dBA}$ (35 dBA above background) tone presented during the entire reinforcement delay after a reward had been earned on the VI $60-\mathrm{sec}$ schedule. The other animals experienced only the $500-\mathrm{msec}$ rein- forcement delay. The response-independent food pellets were not signaled for either group. Training consisted of 20 sessions. Each session lasted $40 \mathrm{~min}$.

\section{Results and Discussion}

The group mean response rates, represented as twosession blocks, can be seen in the left panel of Figure 3. Examination of this figure reveals that, over the first half of training, the signaled group developed a higher response rate than did the group receiving no signal; however, this effect was reversed over the latter half of training. A two-factor ANOVA (signal $\times$ block) conducted upon the response rates depicted in the left panel of Figure 3 revealed a statistically significant interaction of the two factors $[F(9,126)=24.21, p<.001]$. The simple main effects for each of the blocks were analyzed separately, and revealed a significant enhancement of responding by the signal on Blocks 3,4 , and 5 $(p s<.05)$. A significant attenuation of responding was produced by the signal on Blocks 8, 9, and 10 ( $p$ s $<.05$ ). No statistically reliable differences emerged on any other block ( $p s>.10)$.

The right panel of Figure 3 displays the number of responses emitted for the total number of food pellets received (i.e., both response-dependent and -independent). Inspection of these data reveals that the signaled group initially emitted more responses per reinforcer than did the unsignaled group. This pattern of results was later reversed, such that over the latter blocks of training, the signaled group emitted fewer responses per reinforcer. A two-factor ANOVA (signal $\times$ block) conducted upon the efficiency scores depicted in the right panel of Figure 3 revealed a statistically significant interaction of the two factors $[F(9,126)=41.34, p<.001]$. Analysis of the simple main effects revealed that the unsignaled group was

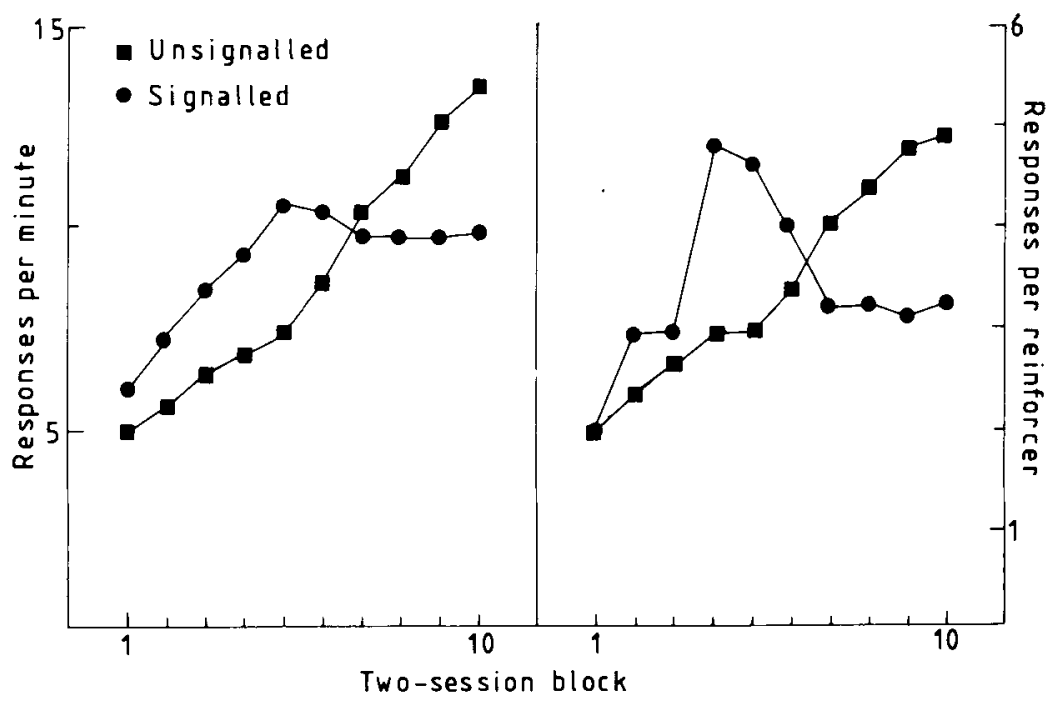

Figure 3. Results from Experiment 3. Left panel: Mean response rates represented as two-session blocks. Right panel: Mean responses per reinforcer represented as two-session blocks. Signalled = groups that received signaled reinforcement, unsignaled = groups that received unsignaled reinforcement. 
more efficient than the signaled group on Blocks 4 and 5 (ps < .05). On Blocks 8, 9, and 10, however, the signaled group was more efficient $(p s<.05)$. No significant difference emerged on any other block ( $p s>10$ ). A highly similar pattern of results emerged when the number of responses emitted for earned reinforcers alone was considered. The biphasic effect was not predicted by the correlation view of signaling effects. This account predicts a signal-induced potentiation of performance with a poor correlation between responding and reinforcement, as was produced by unearned reinforcers. It is worth noting that the present biphasic results may reflect differential effects of the signal on a weak response-reinforcer association early in training and the presumably stronger association late in training (Colwill \& Rescorla, 1988). Although any discussion of the present biphasic effect is clearly post hoc, the results could be seen as consistent with the associative strength account of Schachtman et al. (1987)-that is, overshadowing and potentiation of strong and weak response-reinforcer associations, respectively. However, given the failure of the associative strength theory of signaled reinforcement in accounting for other signal-induced effects such as those obtained in Experiments 1 and 2 and the potentiation on VR schedules with brief signaled delays (Reed et al., 1988a), this explanation is also unlikely. Furthermore, no such biphasic effect has been observed in other situations in which the response-reinforcer association is presumed to be initially weak and later strong (e.g., Schachtman et al. 1987).

The number of reinforcers earned per session was also measured in the present study. Over the first five twosession blocks, the signaled group consistently earned more reinforcers than the the unsignaled group. Over the last five two-session blocks, however, the group means were quite comparable. The collapsed group means for all 10 two-session blocks were 23.0 and 26.0 reinforcers per session for the unsignaled group and signaled group, respectively. A two-factor ANOVA (signal $\times$ block) conducted on the first five two-session blocks revealed a statistically significant interaction of the two factors $[F(9,126)$ $=2.96, p<.01]$. Analysis of the simple main effect of signal over each block revealed that the signaled group earned significantly more reinforcers per session than did the unsignaled group on Blocks 1, 3, 4, and 5 ( $p s<.05$ ). There was no significant difference between the groups on any other block $(p s>.10)$. The present experiment produced a biphasic effect in response rates and efficiency scores. An initial signal-induced potentiation effect early in training gave rise to a signal-induced attenuation of responding.

\section{GENERAL DISCUSSION}

In the present experiments, we attempted to demonstrate the manner in which the correlation between response and reinforcement influences the effects of signaling reinforcement. Schachtman et al. (1987) suggested that when the response-reinforcer association is strong, a signal imposed between the response producing reinforcement and the delivery of reinforcement will attenuate responding. In contrast, when the response-reinforcer association is weak, a signal should potentiate responding. In the present study, we examined a modified version of this view that was aimed at accommodating the signal-induced potentiation of responding noted on VR schedules; that is, when the contingency or correlation between responding and reinforcement is poor, a signal will enhance rates of responding, but when the correlation is strong, responding will be overshadowed by a signal.

Experiment 1 demonstrated instrumental overshadowing and potentiation effects on VI and VR schedules, respectively, when the VR and VI groups were equated for the number of responses emitted per reinforcer. This finding is inconsistent with a correlation account of signaled reinforcement, since the VI and VR groups experienced comparable response-reinforcer correlations and similar reinforcement delays, which should have produced identical signaling effects for the schedules.

In Experiment 2, a signal produced an attenuation of responding on a simple VI schedule; however, when presented on a schedule with a negative feedback function between responding and reinforcement (i.e., a poor correlation was produced between the response and reinforcement due to the large number of nonreinforced responses), the signal had little effect on response rate. In Experiment 3, although an initial signal-induced elevation of responding was obtained on a schedule that produced a poor correlation between responding and reinforcement by the delivery of response-independent reinforcers, the reinforcement signal subsequently came to attenuate responding.

The present results do not support the correlation view of signaled reinforcement effects. Rather, results opposite to those predicted by this view were obtained on many of the schedules in which this notion was explicitly tested (Experiments 1 and 3). The present findings suggest that the response-reinforcer correlation (at least in the direct, simple sense that was examined in these experiments) is not the critical factor underlying such effects. Thus, the sensitivity of signaling effects to factors such as the duration of the reinforcement delay (e.g., Schachtman et al., 1987; Williams \& Heyneman, 1982), interval versus ratio schedules (Experiment 1 above; Reed et al., 1988a; Roberts et al., 1984), schedules that specifically reinforce high (DRH) and low (DRL) rates of responding (Tarpy \& Roberts, 1985), and the strictness of the ratio criterion (Reed et al., 1988a) does not appear to be the result of different response-reinforcer correlations per se, but may reflect the effects of the signal on the structure of behavior and, therefore, the response that makes contact with the reinforcer.

\section{REFERENCES}

Baum, W. M. (1973). The correlation-based law of effect. Journal of the Experimental Analysis of Behavior, 20, 137-155.

Bouton, M. E., Jones, D. L., McPhillips, S. A., Swartzen- 
TRUBER, D. (1986). Potentiation and overshadowing in odor-aversion learning: Role of method of odor presentation, the distal-proximal cue distinction, and the conditionability of odor. Learning \& Motivation, 17, 115-138.

Catania, A. C., \& Keller, J. (1981). The concept of causation. In P. Harzem \& M. Zeiler (Eds.), Advances in analysis of behavior: Vol. 2. Contiguity, contingency, and correlation (pp. 125-167). New York: Wiley.

Colwill, R. M., \& Rescorla, R. A. (1988). The role of responsereinforcer associations increases throughout extended instrumental training. Animal Learning \& Behavior, 16, 105-111.

Dickinson, A., Charnock, D. J. (1985). Contingency effects with maintained instrumental reinforcement. Quarterly Journal of Experimental Psychology, 37B, 397-416.

Dickinson, A., Peters, R. C., \& Shechter, S. (1984). Overshadowing of responding on ratio and interval schedules by an independent predictor of reinforcement. Behavioral Processes, 9, 421-429.

EtTinger, R. H., Reid, A. K., \& Staddon, J. E. R. (1987). Sensitivity to molar feedback functions: A test of molar optimality theory. Journal of Experimental Psychology: Animal Behavior Processes, 13, 366-375.

Gibbon, J., Balsam, P. (1981). Spreading associations in time. In C. M. Locurto, H. S. Terrace, \& J. Gibbon (Eds.), Autoshaping and conditioning theory (pp. 219-253). New York: Academic Press.

Hall, G., Channell, S., \& Schachtman, T. R. (1987). The instrumental overshadowing effect in pigeons: The role of response bursts. Quarterly Journal of Experimental Psychology, 39B, 173-188.

HAMmond, L. J. (1980). The effect of contingency upon appetitive conditioning of free-operant behavior. Joumal of the Experimental Analysis of Behavior, 34, 297-304.

Hammond, L. J., \& Paynter, W. E., JR. (1983). Probabilistic contingency theories of animal conditioning: A critical analysis. Learning \& Motivation, 14, 527-550.

IVERSEN, I. H. (1981). Response interaction with signaled delay of reinforcement. Behavioral Analysis Letters, 1, 3-9.

Kasprow, W. J., Schachtman, T. R., \& Miller, R. R. (1987). The comparator hypothesis of conditioned response generation: Manifest conditioned excitation and inhibition as a function of relative excitatory strengths of CS and conditioning context at the time of testing. Joumal of Experimental Psychology: Animal Behavior Processes, 13, 395-406.

Pearce, J. M., \&all, G. (1978). Overshadowing the instrumental conditioning of a lever press response by a more valid predictor of the reinforcer. Joumal of Experimental Psychology: Animal Behavior Processes, 4, 356-367.
Rachlin, H. A., \& BAUM, W. M. (1972). Effects of alternative reinforcement: Does the source matter? Journal of the Experimental Analysis of Behavior, 18, 231-241.

REed, P., Schachtman, T. R. (1989). Instrumental responding by rats on free-operant schedules with components that schedule responsedependent reinforcer omission: Implications for optimization theories. Animal Learning \& Behavior, 17, 328-338.

Reed, P., Schachtman, T. R., Hall, G. (1988a). Overshadowing and potentiation of instrumental responding in rats as a function of the schedule of reinforcement. Learning \& Motivation, 19, 13-30.

Reed, P., Schachtman, T. R., \& Hall, G. (1988b). Potentiation of responding on a VR schedule by a stimulus correlated with reinforcement: Effects of diffuse and localized signals. Animal Learning \& Behavior, 16, 75-82.

Rescorla, R. A. (1968). Probability of shock in the presence and absence of the CS in fear conditioning. Joumal of Comparative \& Physiological Psychology, 66, 1-5.

RICHARDS, R. W. (1981). A comparison of signaled and unsignaled delay of reinforcement. Journal of the Experimental Analysis of Behavior, 35, 145-152.

RoberTS, J. E., TARPY, R. M., \& LEA, S. E. G. (1984). Stimulusresponse overshadowing: Effects of signaling reward on instrumental responding as measured by response rate and resistance to change. Joumal of Experimental Psychology: Animal Behavior Processes, 10, 244-255.

Schachtman, T. R., Reed, P., \& Hall, G. (1987). Enhancement and attenuation of instrumental responding by signals for reinforcement on a VI schedule. Journal of Experimental Psychology: Animal Behavior Processes, 13, 271-279.

TARPY, R. M., \& RoBERTS, J. E. (1985). Effects of signaled reward in instrumental conditioning: Enhanced learning on DRL and DRH schedules of reinforcement. Animal Learning \& Behavior, 13, 6-12.

Vaughan, W. (1982). Choice and the Rescorla-Wagner model. In M. L. Commons, R. J. Herrnstein, \& H. Rachlin (Eds.), Quantitative analysis of behavior: Vol. 2. Matching and maximizing accounts (pp. 263-279). Cambridge, MA: Ballinger.

Vaughan, W., \& Miller, H. L. (1984). Optimization versus responsestrength accounts of behavior. Journal of the Experimental Analysis of Behavior, 42, 337-348.

WiLliams, B. A., \& Heyneman, N. (1982). Multiple determinants of "blocking" effects on operant behavior. Animal Learning \& Behavior, 10, 72-76.

(Manuscript received February 14, 1989; revision accepted for publication March 19, 1989.) 